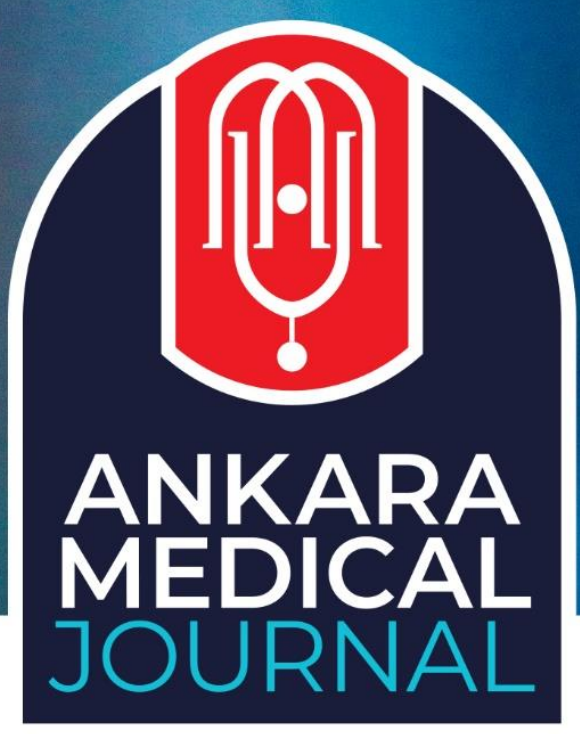

Research Article

Ankara Med J, 2021;(3):484-493 // (10) 10.5505/amj.2021.62347

\title{
PREDICTION OF IN-HOSPITAL MORTALITY IN PATIENTS UNDERGOING ENDOSCOPY FOR NON- VARICEAL UPPER GASTROINTESTINAL BLEEDING
}

\section{(D) Bülent Güngörer ${ }^{1}$}

${ }^{1}$ Ankara City Hospital, Department of Emergency Medicine

Yazışma Adresi / Correspondence:

Bülent Güngörer (e-mail: gungorerbulent@gmail.com)

Geliş Tarihi (Submitted): 18.08.2021 // Kabul Tarihi (Accepted): 08.09.2021

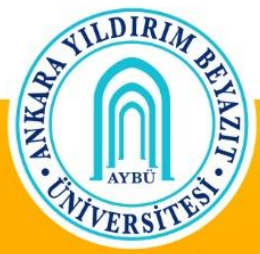

Ankara Yıldırım Beyazıt University Faculty of Medicine 


\begin{abstract}
Objectives: The aim of this study is to investigate the parameters that may contribute to the prediction of inhospital mortality in patients who were admitted to the emergency department with non-variceal upper gastrointestinal system (GI) bleeding and underwent endoscopy.

Materials and Methods: Patients with non-variceal upper GI bleeding who were admitted to the emergency department of our hospital between March 2019 and June 2021 were evaluated retrospectively. Surviving and deceased patients were compared. To predict mortality independently, logistic regression analysis was performed with parameters that were significant.

Results: It was shown that there was a relationship between low albumin and T score, older age, high LDH and higher white blood cell count, and mortality. In the ROC analysis, where the diagnostic accuracy of these five factors in predicting mortality was evaluated, the area under the curve was calculated as 0.84 .

Conclusion: The evaluation of albumin, age, T score, white blood cell and LDH together may be helpful in predicting the in-hospital mortality of patients with non-variceal upper GI bleeding.

Keywords: Upper gastrointestinal bleeding, mortality, T score, nomogram.
\end{abstract}




\section{Introduction}

Gastrointestinal (GI) system hemorrhages constitute a very important group of diseases because of the high frequency of admissions to the emergency department due to it and the fact that it is associated with both morbidity and mortality. ${ }^{1}$ Among GI hemorrhages, upper GI hemorrhages are more common than lower GI hemorrhages. ${ }^{2}$ Its occurrence is twice more in men than in women, and studies report figures of 128 and 65 per 100,000, respectively. ${ }^{3}$ On inspecting the causes, the most common etiology of non-variceal hemorrhages is peptic ulcer, and gastritis, angiodysplasia, Mallory-Weiss syndrome, malignancies, and drug side effects (vitamin K antagonists, etc.) can also be considered. ${ }^{3-5}$

After evaluation in the emergency department, it is decided whether it is related to varicose veins in patients undergoing endoscopy. Non-variceal hemorrhages also constitute an important part of GI hemorrhages. In studies conducted to date, the patient's age, comorbidities, the diagnosis of the underlying hemorrhagic disease, and the drugs used have been related to the prognosis of the patient. ${ }^{3,4,6} \mathrm{It}$ is important to predict the course of the disease in these patients, their mortality, and future complications and inform the patients about them for the effective investigation of the underlying disease and making decisions of follow-up in the hospital. This will prove beneficial in guiding the clinician to predict which patient will be followed up for a short time, which patient will need intensive care, and which patient will need a transfusion and additional treatments.

In light of this information, in this study, we aimed to analyze the clinical and laboratory parameters that can provide information about the problems that may occur in the future in patients with upper GIS hemorrhage who were admitted to the emergency department and investigate whether these parameters can predict the prognosis and mortality of these patients.

\section{Materials and Methods}

Patients with non-variceal upper GI hemorrhage who were admitted to the emergency department and hospitalized for endoscopy between March 2019 and June 2021 were evaluated retrospectively. The clinical and laboratory data of the patients were obtained from the hospital's registration system. During this period, comorbidities, complete blood count parameters (leukocyte and platelet counts, hemoglobin and hematocrit levels, etc.), biochemical markers, kidney and liver function tests, and albumin levels were recorded in patient follow-up forms.

T-score is a clinical scoring system that includes four parameters in which the patient's general condition, heart rate, systolic blood pressure, and hemoglobin levels and it is previously shown to be associated with poor endpoints in cases of upper GI hemorrhages (Table 1). ${ }^{6}$ According to this score, a patient with a poor general 
condition, receives 1 point for each parameter and gets a minimum score of 4 , whereas a patient with a good general condition receives 3 points from each parameter and gets a maximum score of 12 points. The primary endpoint of this study was in-hospital mortality. The deceased and surviving patients were divided into two groups, and their parameters were compared.

\section{Statistical analysis}

All statistical analyses were performed using Stata (version 16.0 MP; StataCorp). The distribution of continuous variables was determined using the Kolmogorov-Smirnov test. Continuous data that showed a normal distribution were presented as mean \pm standard deviation, and the data without a normal distribution were presented as median (range). Categorical data were defined as the number of cases and their percentage. The variables with statistically significant differences and normal distribution were compared.

The Student's t-test was used for two different groups with a normal distribution. The Pearson's chi-square test was used for categorical variables. A univariate logistic regression model was constructed for each variable to show significant predictors of in-hospital causes of mortality, and then those with $\mathrm{p}<0.10$ were tested using a multivariate logistic regression model. The results of multivariate regression analysis were presented as odds ratios of independent predictors of in-hospital mortality and their 95\% confidence intervals.

Receiver operating characteristics (ROC) curve analysis was used to demonstrate the discrimination performance of the final model. Finally, a nomogram containing significant predictors was plotted as a graph. A p-value of $<0.05$ was considered significant in all the statistical analyses.

\section{Results}

During the study period, a total of 82,562 upper endoscopies (all departments including in-patients, outpatients, and emergency department) procedures were conducted in our institute. Eventually, 489 patients who were admitted to the emergency department and underwent endoscopy for upper GI bleeding were included in the study. During this period, 67 patients died while being followed up in the hospital. The mean age of the patients was $64.82 \pm 18.39$ years, and $66.25 \%$ of them were male. On examining the additional morbidities, the most common comorbidities were hypertension (45.81\%), coronary artery disease (34.76\%), and diabetes mellitus (22.08\%). These data are summarized in Table 2. When the surviving and deceased patients were compared, the parameters of age, heart failure, arrhythmia, and malignancy were significantly different between the two groups ( $\mathrm{p}<0.001, \mathrm{p}=0.007, \mathrm{p}=0.042$, and $\mathrm{p}<0.001$, respectively). When the laboratory values and T-scores were compared, significant differences were found between the two groups in terms of urea, alanine aminotransferase, aspartate aminotransferase, gamma-glutamyl transferase, lactate 
dehydrogenase (LDH), and albumin levels; platelet and neutrophil counts; and T-scores (Table 3). According to the multivariate regression analysis, albumin levels, age, neutrophil count, T-scores, and LDH levels were independent predictors of in-hospital mortality (Table 4). In the ROC analysis in which the diagnostic accuracy of these parameters in predicting mortality was evaluated, the area under the curve was found to be 0.84 (Figure 1). The new nomogram scale created using these parameters is presented in Figure 2.

Table 1. T Score parameters and scoring

\begin{tabular}{|l|c|c|c|}
\hline \multirow{2}{*}{ Clinical Parameter } & \multicolumn{3}{|c|}{ Score } \\
\cline { 2 - 4 } & $\mathbf{1}$ & $\mathbf{2}$ & $\mathbf{3}$ \\
\hline General Condition & Poor & Intermediate & Good \\
\hline Heart rate & $>110$ & $90-110$ & $<90$ \\
\hline Systolic blood pressure $(\mathrm{mm} / \mathrm{Hg})$ & $<90$ & $90-110$ & $>110$ \\
\hline
\end{tabular}

Table 2. Basal characteristics and co-morbidities of the patients according to the survival status.

\begin{tabular}{|c|c|c|c|c|}
\hline & $\begin{array}{c}\text { All Patients } \\
\mathrm{N}=489\end{array}$ & $\begin{array}{c}\text { Survivors } \\
\mathrm{N}=422\end{array}$ & $\begin{array}{c}\text { Non-survivors } \\
\mathrm{N}=67\end{array}$ & p-value \\
\hline \multicolumn{5}{|l|}{ Basal Characteristics } \\
\hline Age (SD) & $64.82(18.39)$ & $63.23(18.44)$ & $74.47(15.31)$ & $<0.001$ \\
\hline Male & $324(66.25 \%)$ & $283(67.10 \%)$ & $41(61.20 \%)$ & 0.350 \\
\hline \multicolumn{5}{|l|}{ Co-morbidities } \\
\hline Heart failure & $55(11.25 \%)$ & $41(9.72 \%)$ & $14(20.89 \%)$ & 0.007 \\
\hline Arrhythmia & $88(17.99 \%)$ & $70(16.59 \%)$ & $18(26.87 \%)$ & 0.042 \\
\hline Coronary Artery Disease & $170(34.76 \%)$ & $143(33.88 \%)$ & $27(40.29 \%)$ & 0.310 \\
\hline Chronic Kidney Disease & $51(10.42 \%)$ & $40(9.48 \%)$ & $11(16.42 \%)$ & 0.084 \\
\hline Cerebrovascular Disease & $42(8.58 \%)$ & $35(8.29 \%)$ & $7(10.45 \%)$ & 0.560 \\
\hline Chronic liver disease & $7(1.43 \%)$ & $7(1.66 \%)$ & $0(0 \%)$ & 0.290 \\
\hline Hypertension & $224(45.81 \%)$ & $191(45.26 \%)$ & $33(49.25 \%)$ & 0.540 \\
\hline Diabetes mellitus & $108(22.08 \%)$ & $90(21.33 \%)$ & $18(26.86 \%)$ & 0.310 \\
\hline Gastritis/ulcer & $32(6.54 \%)$ & $29(6.87 \%)$ & $3(4.47 \%)$ & 0.460 \\
\hline Chronic lung disease & $48(9.82 \%)$ & $37(8.77 \%)$ & $11(16.42 \%)$ & 0.051 \\
\hline Malignancy & $58(11.86 \%)$ & $36(8.53 \%)$ & $22(32.84 \%)$ & $<0.001$ \\
\hline
\end{tabular}


Table 3. Baseline laboratory markers of patients according to mortality

\begin{tabular}{|c|c|c|c|c|}
\hline & $\begin{array}{c}\text { All Patients } \\
\mathrm{N}=489\end{array}$ & $\begin{array}{c}\text { Survivors } \\
\mathrm{N}=\mathbf{4 2 2}\end{array}$ & $\begin{array}{c}\text { Non-survivors } \\
\mathrm{N}=67\end{array}$ & p-value \\
\hline Urea, mean (SD), mg/dL & $90.62(63.54)$ & $86.56(60.72)$ & $115.91(74.83)$ & $<0.001$ \\
\hline ALT, mean (SD), U/L & $23.69(38.37)$ & $21.29(20.85)$ & $38.46(89.30)$ & $<0.001$ \\
\hline AST, mean (SD), U/L & $26.41(43.19)$ & $23.8(24.51)$ & $43.83(99.71)$ & $<0.001$ \\
\hline GGT, mean (SD), IU/L & $41.26(76.24)$ & $35.93(64.41)$ & $76.28(123.85)$ & $<0.001$ \\
\hline Amylase, mean (SD), U/L & $64.77(34.86)$ & $64.03(31.55)$ & $70.23(53.49)$ & 0.230 \\
\hline LDH, mean (SD), U/L & $227.40(143.53)$ & $211.01(98.44)$ & $335.67(283.55)$ & $<0.001$ \\
\hline Albumin, mean (SD), g/L & $35.62(6.28)$ & $36.51(5.89)$ & $29.77(6.05)$ & $<0.001$ \\
\hline MPV, mean (SD), fL & $8.44(1.10)$ & $8.31(1.05)$ & $8.60(1.12)$ & 0.096 \\
\hline PDW, mean (SD), fL & $54.20(11.45)$ & $54.08(11.43)$ & $55.22(11.88)$ & 0.460 \\
\hline Platelet, mean (SD), x103/mL & $283.42(144.71)$ & $274.52(121.39)$ & $339.03(239.09)$ & $<0.001$ \\
\hline Neutrophil\#, mean (SD) & $7928.40(4757.93)$ & $7440.33(3992.72)$ & $11002.81(7387.16)$ & $<0.001$ \\
\hline \multicolumn{5}{|l|}{ T Score } \\
\hline 4 & $5(1.10 \%)$ & $4(1 \%)$ & $1(1.70 \%)$ & \multirow{9}{*}{$<0.001$} \\
\hline 5 & $5(1.10 \%)$ & $0(0 \%)$ & $5(8.30 \%)$ & \\
\hline 6 & $15(3.30 \%)$ & $10(2.50 \%)$ & $5(8.30 \%)$ & \\
\hline 7 & $39(8.50 \%)$ & $30(7.50 \%)$ & $9(15 \%)$ & \\
\hline 8 & $71(15.50 \%)$ & $55(13.80 \%)$ & $16(26.70 \%)$ & \\
\hline 9 & $99(21.60 \%)$ & $88(22.10 \%)$ & $11(18.30 \%)$ & \\
\hline 10 & $97(21.10 \%)$ & $88(22.10 \%)$ & $9(15 \%)$ & \\
\hline 11 & $76(16.60 \%)$ & $72(18 \%)$ & $4(6.70 \%)$ & \\
\hline 12 & $52(11.30 \%)$ & $52(13 \%)$ & $0(0 \%)$ & \\
\hline
\end{tabular}

Table 4. Results of multivariable significant predictors

\begin{tabular}{|l|c|c|c|}
\hline & Odds Ratio & 95\% Confidence Interval & p value \\
\hline Albumin & 0.90 & $0.84-0.96$ & 0.002 \\
\hline Age & 1.04 & $1.01-1.06$ & 0.009 \\
\hline Neutrophil & 1.00 & $1.00-1.00$ & 0.004 \\
\hline T-score & 0.79 & $0.63-0.99$ & 0.036 \\
\hline LDH & 1.00 & $1.00-1.01$ & 0.001 \\
\hline
\end{tabular}




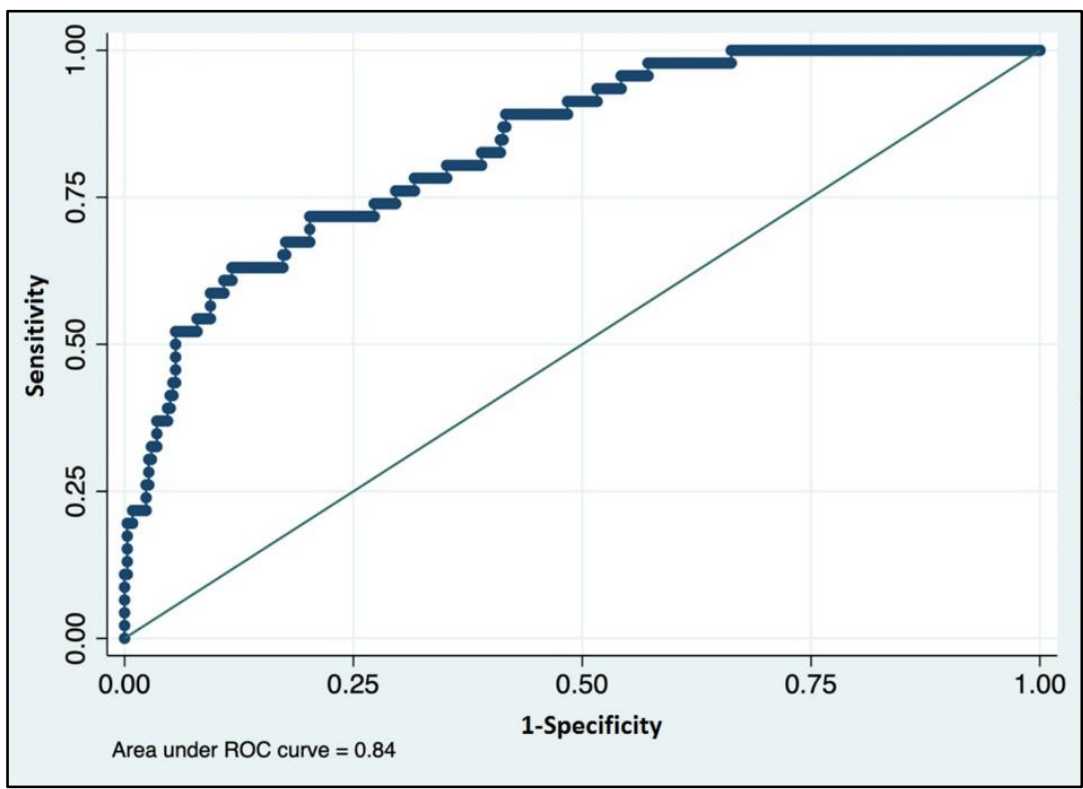

Figure 1. Area under of ROC curve for independently significant predictors of mortality

\section{Nomogram}

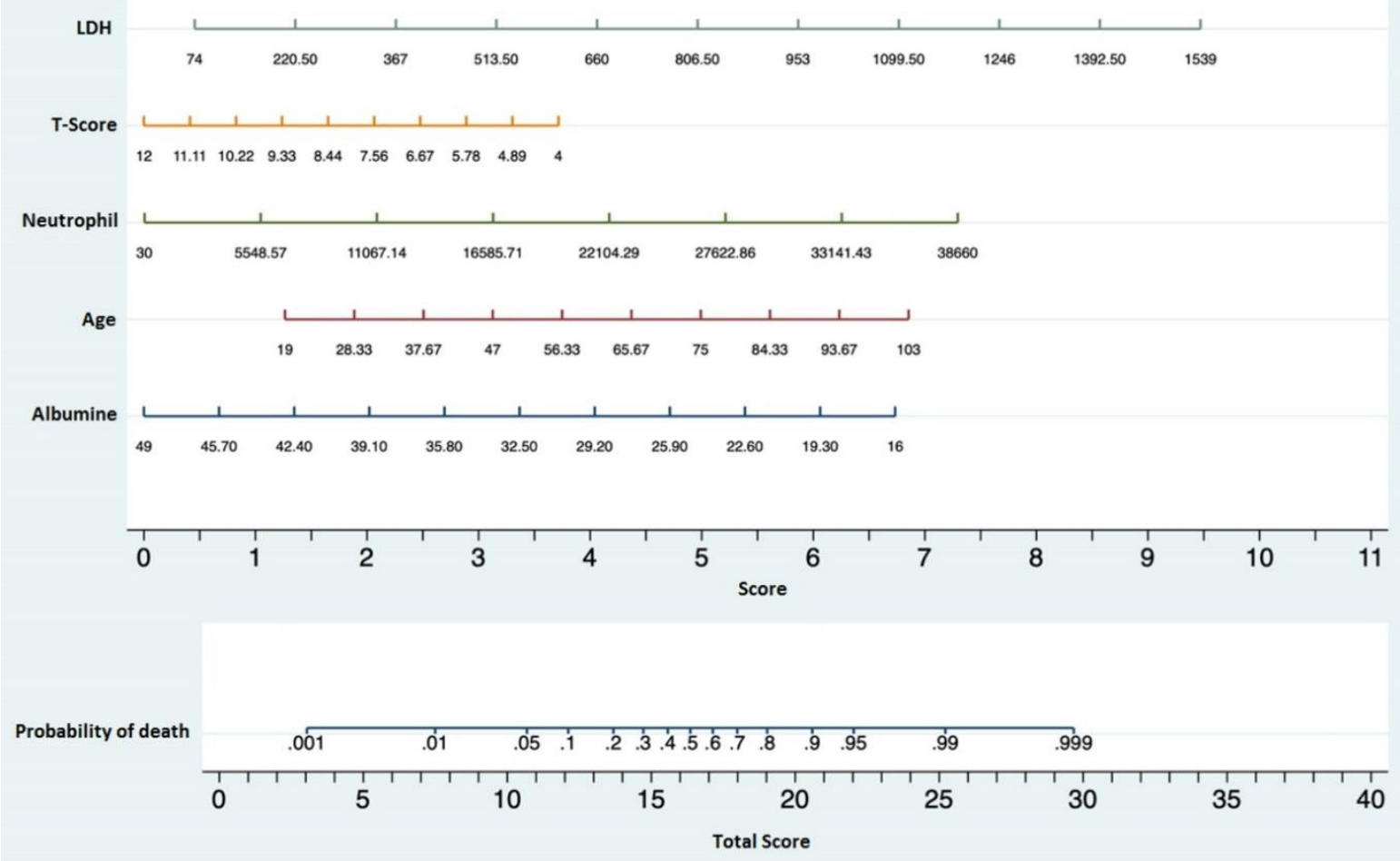

Figure 2. The nomogram of independently significant predictors of mortality 


\section{Discussion}

In this study, we found that the in-hospital prognosis and mortality of patients admitted to the emergency department with non-variceal upper GIS hemorrhage had an inverse relationship with albumin levels and Tscores and a direct relationship with the neutrophil count, age, and LDH levels. Our study is the first to show these relationships, which provides clinicians essential clues for predicting mortality that may develop in the hospital with the help of easily calculable data such as the results of some biochemical analysis and T-score of patients who were admitted to the emergency department with non-variceal upper GIS hemorrhage. Predicting an important outcome such as mortality using the clues related to these types of diseases that are prone to complications and may progress with additional problems will support the clinician who monitors the patient.

On examining the course of GI hemorrhages over the years, it was observed that the incidence and mortality have decreased over the years. This decrease is consistent with the developments in emergency medicine practice and interventional procedures. ${ }^{7}$ Both education provided in the emergency medicine clinics and increased number of trained specialists, as well as the expansion of gastroenterology clinics and specialists in Turkey, has led to improvements in the follow-up and treatment of these patients. In a study by Sezikli et al., non-variceal upper GIS hemorrhages were examined for one year, and ulcer, gastritis, angiodysplasia, and Mallory-Weiss syndrome were found to be the most common causes. ${ }^{8}$

In previous similar studies, the relationship between hematocrit levels and prognosis of patients was investigated, and hematocrit levels $<30 \%$ were associated with poor prognosis in these patients. ${ }^{9}$ In a study by Rao et al., a history of cerebrovascular disease and low albumin levels were found to be associated with 30 -day mortality. ${ }^{10}$ In another study, hemorrhages due to overdose in patients taking warfarin were analyzed retrospectively. The mortality of the patients within 30 days after endoscopy was predicted using simple laboratory parameters of the patient, and low albumin levels, hypertension, alcohol use, and old age were found to be associated with mortality. ${ }^{11}$ Similarly, in a study by Shafaghi et al., low albumin levels were found to be associated with in-hospital mortality. ${ }^{12}$ In our study, similar to the results of these studies, low albumin levels and age were found to be associated with mortality.

Some patients included in the current study might have been using anticoagulant drugs and our results might have been affected by this situation. Although there are controversial findings regarding the impact of these drugs on the clinical outcomes, such as duration of hospital stay and risk of re-bleeding, these drugs may lead to poor prognosis. 13-15 Therefore, a closer follow-up of patients using anticoagulants and antiaggregants is required to improve patient management. In addition, in the current study, we have found that albumin level, T-score, neutrophil count, age, and LDH levels should be taken into account. Using the provided nomogram based on these parameters, patients with a higher risk of mortality could be determined, and applications such 
as earlier endoscopic evaluation, early admission to the intensive care unit and early blood transfusion could be considered for providing better health care.

The fact that the patients were evaluated retrospectively can be considered as a limitation of our study; however, it can be considered that this aspect of the study has been strengthened by the fact that a sufficient number of patients were analyzed and a large number of patients with GIS hemorrhage were admitted to our hospital. Another limitation of this study is that we were unable to compare mortality rates according to the cause of upper GI bleeding due to a lack of data.

In conclusion, in this study, we found that the mortality of patients with non-variceal upper GI hemorrhage who were admitted to the emergency department is inversely related to albumin levels and T-scores and directly related to neutrophil count, age, and LDH levels. We believe that these findings would be helpful for clinicians in predicting the mortality of patients.

\section{Ethical considerations}

This study was confirmed by the local ethics board (Number: E2-21-684) on 14.07.2021.

\section{Conflict of Interest}

The author declares no conflict of interest. 


\section{References}

1. Wuerth BA, Rockey DC. Changing Epidemiology of Upper Gastrointestinal Hemorrhage in the Last Decade: A Nationwide Analysis. Dig Dis Sci. 2018;63(5):1286-93 (doi:10.1007/s10620-017-4882-6).

2. Lanas A, Perez-Aisa MA, Feu F, et al. A nationwide study of mortality associated with hospital admission due to severe gastrointestinal events and those associated with nonsteroidal antiinflammatory drug use. Am J Gastroenterol. 2005;100(8):1685-93 (doi:10.1111/j.1572-0241.2005.41833.x).

3. Enestvedt BK, Gralnek IM, Mattek N, Lieberman DA, Eisen G. An evaluation of endoscopic indications and findings related to nonvariceal upper-GI hemorrhage in a large multicenter consortium. Gastrointest Endosc. 2008;67(3):422-9 (doi:10.1016/j.gie.2007.09.024).

4. Kaya FB, Ozakin E, Coşkun Yüksel G, Karakilic ME, Kaya S, Canakci ME. Use of Prothrombin Complex Concentrate in the Emergency Department for Cases With Warfarin Overdose: A Retrospective Study. Ankara Medical Journal. 2020;20(4):1071-81 (doi:10.5505/amj.2020.82085).

5. Lanas A, Dumonceau JM, Hunt RH, et al. Non-variceal upper gastrointestinal bleeding. Nat Rev Dis Primers. 2018;4:18020 (doi:10.1038/nrdp.2018.20).

6. Tammaro L, Di Paolo MC, Zullo A, et al. Endoscopic findings in patients with upper gastrointestinal bleeding clinically classified into three risk groups prior to endoscopy. World journal of gastroenterology: WJG. 2008;14(32):5046.

7. Loperfido S, Baldo V, Piovesana E, et al. Changing trends in acute upper-GI bleeding: a population-based study. Gastrointest Endosc. 2009;70(2):212-24 (doi:10.1016/j.gie.2008.10.051).

8. Akkan Çetinkaya Z, Sezikli M, Bünül F, Şirin G. A Revision of Patients Who Underwent Gastroscopy Because Of Non-Variceal Upper Gastrointestinal Bleeding In The Last Year. Kocaeli Medical Journal. 2013;2(1):11-4.

9. Balderas V, Bhore R, Lara LF, Spesivtseva J, Rockey DC. The hematocrit level in upper gastrointestinal hemorrhage: safety of endoscopy and outcomes. Am J Med. 2011;124(10):970-6 (doi:10.1016/j.amjmed.2011.04.032).

10. Rao VL, Gupta N, Swei E, et al. Predictors of mortality and endoscopic intervention in patients with upper gastrointestinal bleeding in the intensive care unit. Gastroenterol Rep (Oxf). 2020;8(4):299-305 (doi:10.1093/gastro/goaa009).

11. Tolunay H, Surel AA. An easy nomogram to predict 30-day mortality in warfarin overdose patients undergoing endoscopy for gastrointestinal bleeding. Laparoscopic Endoscopic Surgical Science. $2021 ; 28(1): 29$.

12. Shafaghi A, Gharibpoor F, Mahdipour Z, Samadani AA. Comparison of three risk scores to predict outcomes in upper gastrointestinal bleeding; modifying Glasgow-Blatchford with albumin. Rom J Intern Med. 2019;57(4):322-33 (doi:10.2478/rjim-2019-0016). 
13. Solakoglu T, Koseoglu H, Atalay R, Sari SO, Yurekli OT, Akin E, Bolat AD, Buyukasik S, Ersoy O. Impact of anti-aggregant, anti-coagulant and non-steroidal anti-inflammatory drugs on hospital outcomes in patients with peptic ulcer bleeding. Saudi J Gastroenterol: 2014;20(2):113-9 (doi: 10.4103/13193767.129476).

14. Kawai T, Fukuzawa M, Moriyasu F, Yamashina A. Antithrombotic drug and gastrointestinal injuries. Nihon Rinsho. 2013;71(2):365-8.

15. Di Minno A, Spadarella G, Spadarella E, Tremoli E, Di Minno G. Gastrointestinal bleeding in patients receiving oral anticoagulation: Current treatment and pharmacological perspectives. Thromb Res. 2015;136(6):1074-81 (doi: 10.1016/j.thromres.2015.10.016). 